\title{
PLANTEAMIENTO DE LOS ESTILOS DE ENSEÑANZA DESDE UN ENFOQUE COGNITIVO-CONSTRUCTIVISTA
}

\author{
Approaching teaching styles from a Cognitive Constructivist perspective
}

\author{
Paula Renés Arellano \\ e-mail: paula.renes@unican.es \\ Universidad de Cantabria. España
}

\section{RESUMEN}

Comprender cómo enseña el profesorado es reflexionar sobre su manera de pensar y hacer, y la relación existente entre los procesos que permiten la existencia de una enseñanza y un aprendizaje. En este trabajo se presenta una reflexión teórica sobre las maneras de enseñar del profesorado, sus Estilos de Enseñanza (EdE), sustentada en un enfoque cognitivoconstructivista que tiene como referentes unos determinados comportamientos y Estilos de Aprendizaje (EdA). Para ello, se expone un modelo teórico sobre los EdE desde una visión cognitiva en la que el comportamiento está influenciado por diversas variables que lo determinan, como las características e intereses personales o el contexto sociocultural. Finalmente, se enfatiza la necesidad de que la enseñanza y los EdE estén guiados por procesos de reflexión y análisis de uno mismo y de las realidades que la constituyen, teniendo presente cómo y de qué manera se actúa con los demás y con los agentes educativos con los que se comparte diariamente la tarea docente.

PALABRAS CLAVE: estilos de enseñanza; estilos de aprendizaje; educación; enseñar; aprender.

\begin{abstract}
Understanding how teachers teach is to reflect on their ways of thinking and doing, and the relationship between the processes that allow the existence of teaching and learning. In this paper we present a theoretical reflection on the teaching methods of teachers, their Teaching Styles (EdE), based on a cognitive-constructivist approach that has as reference certain behaviors and Learning Styles (EdA). For this, a theoretical model is presented on the EdE from a cognitive view in which the behavior is influenced by several variables that determine it, such as personal characteristics and interests or the sociocultural context. Finally, it is emphasized the need for teaching and EHEs to be guided by processes of reflection and analysis of oneself and the realities that constitute them, keeping in mind how and in what way one acts with others and with educational agents with Which are shared daily the teaching task.
\end{abstract}

KEY WORDS: teaching styles; learning styles; education; teaching; learning.

Recibido/Received: 30/07/2017

Aprobado/Aproved: 23/12/2017

Cómo referenciar este artículo / How to reference this article:

Renés Arellano, P. (2018). Planteamiento de los estilos de enseñanza desde un enfoque cognitivo-constructivista. Tendencias Pedagógicas, 31, 47-68. doi: http://dx.doi.org/10.15366/tp2018.31.002 


\section{INTRODUCCIÓN}

Las diversas acepciones que han ido surgiendo en torno a la enseñanza y sus modelos no pueden entenderse al margen del contexto social e histórico en el que la sociedad ha ido evolucionando. El proceso educativo en el que la enseñanza y el aprendizaje se hacen evidentes, en los que la intencionalidad y el ser consciente de aquello que se adquiere retorna, en ocasiones, como algo inverosímil solo puede entenderse desde la planificación, sistematización y adaptabilidad a las realidades docentes en las que está adscrito.

El origen de la educación proviene de dos etimologías, educare, que significa alimentar o llenar de conocimientos, y educere que se corresponde con la capacidad del alumno de potenciar su aprendizaje; la enseñanza, originaria del latín insignare, es decir, "señalar hacia", "orientar hacia", entiende que el docente, se convierte en este proceso en un guía, un orientador que puede conducir al alumnado hacia la sabiduría.

Sin embargo, en la práctica docente ¿existe realmente una diferencia entre la enseñanza tradicional y la moderna? En este sentido, se puede cuestionar si verdaderamente se ha superado una enseñanza caracterizada por un estilo convencional, sistemático y formal, en el que las normas ya están establecidas y los criterios son comunes a todo el alumnado, en el que prima la moral heterónoma y por tanto, en la que la voluntad del alumnado no está sujeta al propio alumno sino que ya viene predeterminada. Habría que plantearse si en estos momentos se tiene presente al alumno y su grupo como sujetos autónomos y a la vez, interrelacionados. En cierto modo, que actualmente las nuevas políticas y prácticas educativas se han centrado en el alumno, en lo que le rodea, su individualidad y su carácter social, pero la cuestión es si los docentes son capaces de responder a esa individualidad y si tienen las herramientas que les permitan adaptarse a estas realidades. Es por ello, que los EdA (Estilos de Aprendizaje) y los EdE (Estilos de Enseñanza) que se describen en este trabajo, intentan dotar de autonomía al profesor a la hora de establecer metodologías docentes que le ayuden a acercarse a la realidad de su alumnado, comprenderlo y motivarlo para que logren un aprendizaje significativo.

Por ende, se necesita que el docente, guía en el proceso de enseñanza, oriente al educando hacia el desarrollo holístico, potenciando el protagonismo de este y su desarrollo autónomo. Este estilo progresista, enfrentado al tradicional, entiende la educación y la enseñanza desde una visión abierta, constructiva e interrelacionada, donde la imaginación y la creatividad cobran sentido, en la que la moral heterónoma permite al alumnado establecer sus propios criterios y al docente favorecer enseñanzas adaptadas a la diversidad del alumnado.

\section{CONTEXTUALIZACIÓN DE LOS ESTILOS DE ENSEÑANZA (EDE)}

\subsection{La enseñanza de ayer y de hoy}

La imagen del docente así como su acción, la enseñanza, se ha visto transformada en función del momento histórico-social. Dependiendo de las estructuras sociales, la enseñanza ha pasado de ser considerada pasiva a 
transformase en un proceso activo. Ya en la Edad Antigua (siglo $\mathrm{V}$ a. c.), la enseñanza recaía sobre el maestro, el paidogogo, aquella persona que se encargaba de acompañar al niño en su aprendizaje. Filósofos y pensadores del momento como Platón y Aristóteles se preocuparon de la educación, de buscar aquellos métodos que mejor se adecuaban a su alumnado. Los dos concebían que la educación debía ser una prioridad del estado, del gobierno, y que todo aquello que se gestase en él influiría positiva o negativamente en la educación y en consonancia, en el proceso de enseñanza. No será hasta la Edad Moderna cuando la enseñanza, aun siendo magiocéntrica, unidireccional y pasiva, inicie un periodo de transformación, en el que pedagogos relevantes como Comenio, Erasmo de Rotterdam o Vives empiezan a plantearse cómo se puede enseñar, qué contenidos son relevantes para educar al ciudadano de la época y qué conocimientos debe que adquirir el maestro para estar cualificado profesionalmente. Será, en la edad contemporánea (siglo XVIII-XX) cuando el maestro adquiere mayor protagonismo, cuando las escuelas empiecen a ser sinónimo de progreso y cuando surjan en Estados Unidos las primeras instituciones educativas, aunque el papel de la enseñanza continuaría siendo unidireccional.

La enseñanza del siglo XIX se centraba en la instrucción básica, en procesos de alfabetización y habilidades aritméticas, donde la lectura, la escritura y la aritmética eran la base de los programas educativos. En ese momento la formación del maestro no era relevante y por tanto, para enseñar no se precisaba ninguna preparación específica. Concretamente, el papel docente en ese momento, recaía sobre la mujer joven como la persona que se encargaría de "cuidar" la escuela (Arends, 2007). Sería ya a principios del siglo XX, cuando la educación no recaía solamente en conocimientos básicos sino que los cambios sociales e históricos como la llegada de inmigrantes, la migración de las personas de áreas rurales a urbanas, o la transición de la vida familiar a la laboral, suscitó la necesidad de reestructurar el programa educativo e incorporar conocimientos sobre la salud, la preparación vocacional o la ética social.

En Estados Unidos se crearon escuelas especiales para capacitar al profesorado en materias específicas y para garantizar una formación pedagógica necesaria en las instituciones educativas. De hecho, los maestros debían formarse dos años en la universidad y a mediados del siglo XX, la mayoría había adquirido titulaciones de licenciatura. Concretamente, en 1834, surge en España la primera Escuela Normal de Maestros en Madrid, la primera institución oficial de maestros de enseñanza primaria, que posteriormente se extendieron por todo el panorama nacional. En todo este transcurso se inicia una transformación de la enseñanza tradicional, afincada en una pedagogía piramidal sustentada en el autoritarismo y la memorización, hacia un nuevo modelo de pedagogía en la que la bidireccionalidad alumno-maestro sea el pilar que, actualmente, se mantiene en las aulas. "El arte de enseñar" (González-Pérez, 1993:139) genera que la enseñanza busque nuevos modos de expresarse, entenderse y desarrollarse. Autores como Manjón, Montessori, Freire o Makarenko entre otros, propugnan modelos de enseñanza diversos, centrados en el proceso de autonomía y desarrollo integral del alumno.

Siguiendo el hilo conductor de la enseñanza, su origen y evolución, se hace necesaria una aproximación etimológica. Enseñar es crear las 
condiciones para que los esquemas de conocimiento que construye el estudiante sean correctos, ricos y variados. La enseñanza-aprendizaje es incompatible con la enseñanza como una transmisión de conocimientos unidireccionales, ambos procesos deben ir de la mano, ser bidireccionales si se desea llegar a una calidad educativa óptima. Algunos autores (Zabalza, 2006; Colén, Giné e Imbernon, 2006; Salinas y Urbina, 2006) coinciden en que la enseñanza debe responder al aprendizaje desde su vertiente social, constructiva, significativa y autónoma.

La enseñanza se considera una actuación organizada y deliberada, en la que el docente utiliza los métodos adecuados para promover el aprendizaje del alumnado (Haidt, 2000). Debe generar en el estudiante interés y necesidad de aprender, además de conocimientos, a través de los cuales la persona sea "consciente de que la educación es la base del desarrollo personal, social, profesional..." (Sánchez-Ortega, 2011:11). En este proceso de enseñanza es necesario que el aprendizaje se mantenga activo, constructivo, organizado y dinámico, que no sea solamente un producto, sino el resultado inacabado de un proceso continuo. El aprendizaje será activo cuando el estudiante sea capaz de realizar actividades de procesamiento o de creación de la información que le hagan competente y autónomo, porque él es quien aprende, el protagonista de su propio aprendizaje. Será, a su vez, constructivo, porque el aprendizaje solamente puede entenderse desde un proceso de construcción en el que el estudiante interacciona con los conocimientos previos y nuevos, la información y los estímulos, incentivado por el contexto social, comunicativo y experiencial. Organizado, ya que el aprendizaje requiere de una distribución de conocimientos que permita la adquisición de destrezas de forma gradual. Dinámico, porque el aprendizaje se construye en el proceso de intercambio de información con los demás en el que se modifican las estructuras cognitivas de las personas, se adaptan, y crean nuevos conocimientos que facilitan la interpretación de la realidad.

Por tanto, si el aprendizaje responde a estos términos, la enseñanza también debe adecuarse a cada una de las características intrínsecas a su definición. De esta manera, enseñar se convertirá en un proceso en el que el docente debe cuestionarse cuestiones tales como las siguientes (Gros, 1997 y Colén, Giné e Imbernon, 2006): cómo se establece la relación entre los conocimientos anteriores y las nuevas informaciones que se transmitirán al alumnado, y qué estrategias cognitivas y metacognitivas se están desarrollando actualmente; cuál es la manera más eficaz de organizar los conocimientos por parte del estudiante; qué tipo de actividades, globales y contextuales, favorecerán dichos aprendizajes (tareas complejas y completas); cómo dinamizar el proceso de aprendizaje para lograr que todo el alumnado adquiera las herramientas necesarias que le faciliten ser competente y cuándo y de qué manera se podrá evaluar el proceso de aprendizaje.

La respuesta a las cuestiones planteadas sobre la enseñanza y sus modos de entenderla, es como en el caso del aprendizaje, compleja. Dependiendo del enfoque, el modelo y el paradigma que se considere objeto de análisis, descripción e interpretación, la enseñanza podrá abordarse desde diversas vertientes: cognitivista, constructivista... En este sentido, en el apartado siguiente, se expondrá el modelo seleccionado en este trabajo: una interpretación sustentada en el comportamiento docente y los EdE desde una 
visión cognitivista, en la que el comportamiento está influenciado, no solamente por la variable ambiental, sino también, por otras que lo determinan, como las características e intereses personales.

\subsection{Definición de los Estilos de Enseñanza (EdE)}

Contextualizar los EdE precisa entender qué son los comportamientos y cómo estos favorecen la construcción de dichos estilos, tal y como, recientemente, se ha dejado patente (Renés, Echeverry, Chiang, Rangel y Martínez-Geijo, 2013). El concepto de comportamiento, ya en el siglo XVIII, estaba ligado genéricamente al modo o la forma de actuar o de reaccionar de una persona ante determinadas situaciones, al conjunto de modales inspirados en el estudio de normas conductuales.

El origen del término de comportamiento surgió vinculado a las ramas científicas de la psicología general y experimental, y posteriormente se ha ido incorporando al lenguaje de la pedagogía. El paradigma adoptado en este estudio se sustenta en el modelo cognitivista, desde el cual el comportamiento es entendido como un producto fruto del contexto y que, por tanto, está determinado por otro tipo de factores que lo determinan como pueden ser: el valor que le otorga una persona al objetivo alcanzable o a las aspiraciones e intereses que pueda tener.

En este sentido, el comportamiento es entendido como el resultado que surge de la interacción entre la persona y el ambiente y está determinado por la acción simultánea de todas las variables presentes en las diferentes situaciones de la persona, definición adoptada y compartida por Martínez-Geijo (2002), de quien se ha seguido la investigación planteada en este trabajo; y Fuentes y Espinoza (2014). Por tanto, la enseñanza y los EdE, están constituidos por los diferentes comportamientos que pueden caracterizar a la persona, incluyendo las variables y el contexto que lo determinan. Es decir, que enseñar, como acción consciente, requiere de un proceso de reflexión y análisis de uno mismo y de las realidades que la constituyen, teniendo presente cómo y de qué manera se actúa con el estudiante y con los agentes educativos con los que se comparte diariamente la tarea docente.

Desde esta visión, no se puede ignorar la evidencia de que cada persona es diferente, única y emocional. Tanto los aspectos personales como ambientales influyen en su proceso de aprendizaje, tal y como se ha defendido anteriormente, y por tanto, el docente debe favorecer el desarrollo de la capacidad creativa del alumnado, guiándole y orientándole en su aprendizaje. Por ende, los comportamientos de enseñanza serían la concreción de aquellas acciones que desempeña el profesor en interacción con el ambiente educativo y social, influido por su propia intencionalidad y por el enfoque de enseñanza, pudiendo entenderse solamente, desde el compromiso exitoso entre el que enseña y el que aprende (Martínez-Geijo, 2002).

En esta contextualización los comportamientos de enseñanza se convierten, por tanto, en un engranaje en el que están implicados los valores y actitudes del profesor, su manera de concebir la enseñanza, los conocimientos que se transmiten y el contexto social, educativo y cultural en el que se desarrolla su labor educativa, es decir, responden a una manera de enseñar. 
Por consiguiente, los profesores en su labor de enseñar, deben ser conscientes y responsables de que sus comportamientos influirán en el proceso de aprendizaje de su alumnado, porque son considerados inherentes a la propia enseñanza, y por ello, el docente debe cuidar cómo y de qué manera piensa y actúa en el aula, intentado adaptarse a las realidades individuales, grupales y contextuales. Por tanto, los estilos, definidos anteriormente y compartidos (Alonso, Gallego y Honey, 2012) como el modo o la manera de actuar de una persona en función del entorno y la situación, se tornan en la enseñanza, como los Estilos de Enseñanza (EdE). Porque todo maestro, profesor o docente adopta, como persona, maneras diferentes de comportarse, de actuar, de enseñar. Es su manera de organizar los tiempos, los recursos, de seleccionarlos, interactuar, "hacer enseñanza", lo que determina un tipo de EdE u otro, generando así diferentes categorías de comportamientos y de estilos de enseñanza.

Como diría Freire (1979:55), la gran cantidad de programas educativos que han fallado ha sido debido a que "sus realizadores partieron de su visión personal de la realidad, porque no tomaron en cuenta, aunque fuese por un mínimo instante, al hombre en "situación" a quién se dirigía su programa". Y en concreto, uno de los profesores que respondieron a la cuestión sobre el problema de los programas educativos señalaba en esta misma publicación, "su lenguaje no sintoniza con la situación concreta del hombre concreto a quien hablan. Y su habla es un discurso alienado y alienante". Ya que, como afirman Nortes-Martínez y Nortes-Checa (2012) cuanto más duraderos y transferibles son los resultados de un aprendizaje, se considera más eficaz la enseñanza, y con ella las capacidades adquiridas permanecen y permiten la adquisición de otras.

En esta línea, el modelo que se adopta para definir una enseñanza (el enfoque de enseñanza), está estrechamente relacionado con la manera en la que el docente enseña partiendo del enfoque de enseñanza seleccionado, es decir, el EdE. La eficacia de ese proceso de enseñanza y aprendizaje estará mediatizado por el tipo de enfoque de enseñanza que el docente decida utilizar en función de su alumnado y del contexto, y por el EdE que guía su práctica educativa. Concretamente, investigaciones recientes se han centrado en desarrollar instrumentos de medida sobre dichos enfoques (Salvador, Argos, Ezquerra, Osoro y Castro, 2011; Biggs, Kermberg y Leurrng, 2001 y Gargallo et al., 2010). Sin embargo, mientras que para algunos autores existe una diferenciación entre los enfoques de enseñanza y los EdE, otros investigadores asumen que no existe diferencia en la definición de ambos conceptos (Hervás, 2003).

La diversidad en la tipología de los enfoques de enseñanza ha generado la selección según los intereses de este trabajo, por un lado, el enfoque tradicional y por otro, el enfoque constructivista (Martínez-Geijo, 2008), pero también se pueden recoger otros tales como el tecnológico y el espontaneísta (Gimeno y Pérez-Gómez, 1998). El primero de ellos, se determina por las características señaladas anteriormente sobre la enseñanza tradicional, mientras que el segundo enfoque, también descrito, corresponde con la enseñanza actual, cada vez más activa e integradora. La eficacia de la metodología docente estará determinada, entre otros factores, por el tipo de enfoque de enseñanza que adopte el profesor, porque cada uno de dichos 
enfoques está vinculado a un tipo de aprendizaje o a otro, cada uno de ellos con sus aspectos favorables o inconvenientes (véase figura 1). Se evidencia, a partir de los enfoques, que el papel de los EdE se verá determinado, a su vez, por el tipo de enfoque que se elija en la práctica docente.

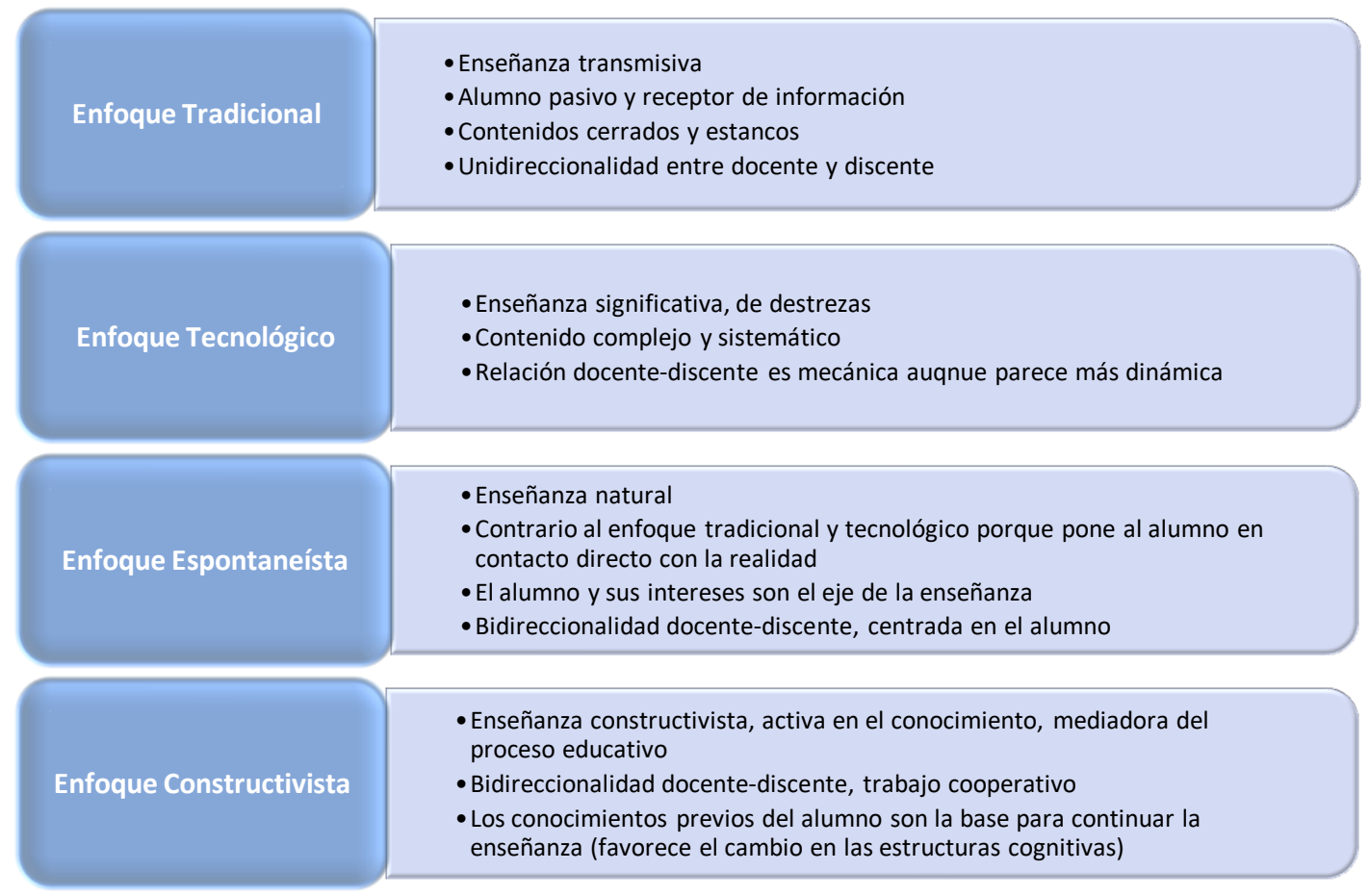

Figura 1. Enfoques de enseñanza: sus características

Con la finalidad de contextualizar qué son los EdE se han seleccionado algunas de las aportaciones presentes en diversas publicaciones y se ha considerado, asumido y compartido la defendida por Martínez-Geijo (2011: 403): los EdE son aquellas categorías de preferencia y comportamientos de enseñanza que

- el docente exhibe habitualmente en cada fase o momento de la actividad de enseñanza

- se fundamenta en actitudes personales que le son inherentes

- han sido abstraídos de su experiencia académica y profesional

- no dependen de los contextos en los que se muestran

Se añade, que dichas categorías tienen como referente los EdA. Por lo que esta definición implica:

a) Establecer criterios para categorizar preferencias y comportamientos de enseñanza.

b) Que el docente los exhiba habitualmente. Es decir, que se encuentren incorporados a sus rutinas y se muestren en el modo particular de actuar en cada momento del proceso de enseñanza.

c) Fundamentarse "o tener su origen" en actitudes personales.

d) Que se encuentren arraigados en su experiencia académica y profesional. 
e) Tener como referencia los EdA.

Desde esta perspectiva (Martínez-Geijo, 2007), asumida y compartida en este trabajo, al igual que en los EdA, cada docente no posee unos EdE puros en relación con las categorías establecidas. Si no que puede manifestar comportamientos que pertenezcan a cada uno los cuatro estilos establecidos, tal y como se estudiará en la fase empírica.

Resulta evidente que la atribución de unos determinados EdE a un docente, debe surgir de un análisis previo y exhaustivo de los comportamientos y preferencia de éste categorizados para tal fin y considerados como los más frecuentes y permanentes en su trayectoria profesional, independientemente del contexto en el que se desenvuelva.

En este sentido, lo EdE son definidos desde diferentes visiones:

González-Peiteado, López-Castedo y Pino-Juste (2013:2) aluden a los EdE como "la tendencia docente de adoptar un determinado modo de interactuar con el alumno en función de las demandas específicas de la tarea, de percibir las necesidades, intereses, capacidades del alumno y de pensar acerca de su praxis educativa".

Isaza y Henao (2012:135) señalan que el EdE "es la forma particular que presenta un docente al momento de organizar la clase y de interactuar con los estudiantes".

A tenor de las aportaciones citadas y otras como las de Contreras (1998), Díaz-Barriga y Hernández-Rojas (2002) y Manterola (2002), el EdE es considerado, por tanto, como el conjunto de comportamientos que adopta el docente en interacción con su alumnado y el contexto que le rodea, respondiendo de una determinada manera o forma ante las situaciones y mediatizado por el enfoque de enseñanza que desee utilizar en su praxis educativa.

\section{MODELO DE CLASIFICACIÓN DE LOS ESTILOS DE ENSEÑANZA (EDE)}

\subsection{Referentes para la construcción de un modelo de EdE}

Las continuas investigaciones sobre los EdA y EdE han generado la necesidad de responder pedagógicamente a los modos de aprender y de enseñar. De tal manera, que algunas instituciones de educación superior muestran a docentes y discentes, a través de sus centros de formación, herramientas, estrategias, instrumentos y posibilidades didácticas para mejorar los procesos de enseñanza y aprendizaje a través de los diferentes estilos.

Concretamente, la Universidad del Norte de Carolina (Greensboro) ha publicado en sus programas académicos del portal online de la institución un apartado específico sobre "Teaching and Learning Styles" ${ }^{1}$, Estilos de Enseñanza y de Aprendizaje. Mientras que indican que no existen investigaciones concluyentes sobre la eficacia de actividades de enseñanza y aprendizaje en función de únicamente un estilo o modelo, sí realzan que estos estudios demuestran que son útiles para identificar y reflexionar sobre la forma

\footnotetext{
${ }^{1}$ Teaching and Learning Styles. Recuperado de https://www.uncg.edu/hhs/oaa/tl_styles.html
} 
de enseñar y de aprender, favoreciendo la creación de una filosofía de enseñanza. Asimismo, se indica una referencia al libro "How People Learn" (Bransford, 2000), en el que se indica que la enseñanza puede favorecer en el estudiante el desarrollo de habilidades para tomar el control de su propio aprendizaje, lo que justifica, la necesidad de utilizar instrumentos y modelos de estilos de enseñanza y aprendizaje por parte de docentes y discentes.

En esta línea, el Centro de Enseñanza y Aprendizaje, Center for Teaching and Learning, de la Universidad de California (Berkeley), aboga por la necesidad actual de prestar mayor interés a los EdE (escuchar, ver, hacer...), en la preparación de la docencia universitaria ${ }^{2}$. Aunque destacan la realidad compleja de las aulas de educación superior, en las que no es fácil adaptar las metodologías y las clases (prácticas o magistrales) a todos y cada uno de los alumnos. Sí se enfatiza que es posible programar actividades que respondan a todos los EdE y así, poder dar respuesta a las realidades individuales y grupales del alumnado. Para lograr este objetivo, y de la misma manera que en el caso anterior, se puede consultar en su página web recursos e instrumentos sobre EdE y EdA. También, la Universidad de Michigan, a través de su centro de formación, Center for Research on Learning and Teaching, ofrece un espacio online (http://www.crlt.umich.edu/tstrategies/tsts) con inventarios y sugerencias sobre cómo dar respuesta a las necesidades de aprendizaje del alumnado.

Algunos de los instrumentos se muestran a continuación (véase tabla 1), destacando que para este estudio se utilizará el instrumento y modelo aportado por Bennett (1979) así como el que planteó Martínez-Geijo (2002) sobre la relación de los comportamientos y los EdE en función de los EdA de Alonso, Gallego y Honey (2012). De manera que, en este trabajo, se está avanzando para ofrecer a la comunidad educativa un modelo pedagógico y un instrumento de evaluación y diagnóstico fiable y válido, que favorezca la identificación de los EdE del docente en función de los EdA de su alumnado. Esto se logrará si se parte de un modelo ya creado y afianzado en la comunidad científica sobre EdA, como es el CHAEA y sus cuatro EdA (Activo, Reflexivo, Teórico y Pragmático), para lograr identificar los cuatro EdE que favorecerán los estilos del alumnado.

\begin{tabular}{|c|c|c|}
\hline AUTOR & INSTRUMENTO & CARACTERÍSTICAS \\
\hline $\begin{array}{c}\text { Angelo y Cross } \\
(1993)\end{array}$ & $\begin{array}{l}\text { Inventario de objetivos de } \\
\text { enseñanza } \\
\text { (TGI-Teaching Goals } \\
\text { Inventory) }\end{array}$ & $\begin{array}{l}\text { Es una autoevaluación de metas de enseñanza } \\
\text { Consta de } 53 \text { preguntas } \\
\text { Los objetivos de enseñanza se dividen en cinco grupos: } \\
\text { habilidades superiores, habilidades básicas, habilidades } \\
\text { específicas, preparación profesional o desarrollo personal. }\end{array}$ \\
\hline $\begin{array}{c}\text { Delgado, Medina y } \\
\text { Viciana } \\
(1996)\end{array}$ & $\begin{array}{l}\text { Questionnaire } \\
\text { (DEMEVI) }\end{array}$ & $\begin{array}{l}\text { Identifica los EdE en función de la forma de intervenir del } \\
\text { docente en la clase. } \\
\text { Consta de } 60 \text { preguntas ( } 10 \text { por cada EdE) } \\
\text { Identifica EdE: tradicionales, individualizadores, participativos, } \\
\text { socializadores, cognoscitivos y creativos. }\end{array}$ \\
\hline $\begin{array}{l}\text { Grasha } \\
\text { (1996) }\end{array}$ & $\begin{array}{l}\text { Cuestionario de EdE } \\
\text { (Teaching Style) }\end{array}$ & $\begin{array}{l}\text { Identifica técnicas de enseñanza y con ello, el papel del } \\
\text { profesor para los respectivos EdE. } \\
\text { Consta de } 40 \text { preguntas } \\
\text { Identifica } 5 \text { EdE: experto, autoridad formal, modelo personal, }\end{array}$ \\
\hline
\end{tabular}

\footnotetext{
${ }^{2}$ Center for Teaching and Learning. Recuperado de http://teaching.berkeley.edu/learning-styles
} 


\begin{tabular}{|c|c|c|}
\hline & & facilitador y delegador \\
\hline Pratt (1998) & $\begin{array}{l}\text { Inventario de perspectivas de } \\
\text { enseñanza-IPE } \\
\text { (TPI-Teaching Perspectives } \\
\text { Inventory) }\end{array}$ & $\begin{array}{l}\text { Identifica perspectivas del docente: qué creen, logran y } \\
\text { hacen. } \\
\text { Consta de } 75 \text { ítems } \\
\text { Identifica } 5 \text { perspectivas del profesor: Transmisivo, } \\
\text { aprendizaje, de desarrollo, cultivar, reforma social. }\end{array}$ \\
\hline Bennett (1979) & Cuestionario del maestro & $\begin{array}{l}\text { Consta de } 28 \text { ítems } \\
\text { Identifica } 12 \text { EdE que se repartirían en } 3 \text { bloques, los Liberal- } \\
\text { Progresista, los Tradicionales-Formales y el resto que se } \\
\text { encuentran en medio de ambos. }\end{array}$ \\
\hline $\begin{array}{l}\text { Martínez-Geijo } \\
\quad(2007)\end{array}$ & $\begin{array}{l}\text { Cuestionario de Estilos de } \\
\text { Enseñanza (CEdE)-España }\end{array}$ & $\begin{array}{l}\text { Diseñado en función del cuestionario CHAEA de EdA de } \\
\text { Alonso, Gallego y Honey (1994) } \\
\text { Consta de } 40 \text { ítems } \\
\text { Identifica comportamientos de enseñanza y EdE }\end{array}$ \\
\hline Chiang et al. (2013) & $\begin{array}{l}\text { Cuestionario Estilos de } \\
\text { Enseñanza (CEE)-Chile }\end{array}$ & $\begin{array}{l}\text { Sustentado en el cuestionario de Martínez-Geijo (CEE) en } \\
\text { lengua española-chilena } \\
\text { Consta de } 71 \text { ítems } \\
\text { Identifica los mismos } 4 \text { EdE del CEE original }\end{array}$ \\
\hline $\begin{array}{l}\text { Renés-Arellano } \\
\text { (2014) }\end{array}$ & $\begin{array}{l}\text { Cuestionario de Estilos de } \\
\text { Enseñanza (CEdE)-España }\end{array}$ & $\begin{array}{l}\text { Diseñado en función del cuestionario CHAEA de EdA de } \\
\text { Alonso, Gallego y Honey (1994) y del CEE de Martínez-Geijo } \\
\text { (2007) } \\
\text { Consta de } 80 \text { ítems } \\
\text { Identifica } 4 \text { EdE: Abierto, Funcional, Estructurado y Formal }\end{array}$ \\
\hline
\end{tabular}

Tabla 1. Instrumentos de diagnóstico sobre EdE.

Las aportaciones de Bennett (1979) y Martínez-Geijo (2007) son los dos modelos educativos sustentados en los EdE que sirven de referente y son el pilar del diseño y desarrollo de este trabajo.

Las razones por las que se ha optado por ello, se basan en que el modelo planteado por Bennett (1979), se sustenta en identificar los EdE a partir de los existentes (teniendo en cuenta los comportamientos de enseñanza), la relación entre dichos estilos y los resultados académicos; tres ámbitos de investigación que se han seguido en este trabajo. La elección del modelo de Martínez-Geijo (2007) ha estado derivada de su continuación a la línea de trabajo de Bennett en cuanto a la identificación de EdE y la relación entre ellos partiendo de los comportamientos de enseñanza, avanzando en el establecimiento de un modelo que partiese de una relación entre EdA y EdE, para lo cual se sirvió del CHAEA (Cuestionario de EdA. Alonso, Gallego y Honey, 1994).

Tomando en consideración las aportaciones de Bennett y Martínez-Geijo, cabe señalar que algunos autores como Hervás (2003), Chiang et al. (2013) y Echeverry (2013), entre otros, han planteado investigaciones similares. A modo de referencia, Hervás (2003) publicó un estudio sobre EdE sustentado en las aportaciones sobre EdA de Gregorc (1984) identificando los estilos Concreto, Secuencial, Aleatorio y Abstracto con habilidades de mediación. Chiang et al. (2013) han presentado la validación adaptada al ámbito chileno del Cuestionario de Estilos de Enseñanza (CEE) de Martínez-Geijo en el que han mantenido los cuatro EdE y modificado la redacción y el número de ítems del instrumento. Concretamente, se han reelaborado algunos ítems para hacerlos 
más compresibles y fáciles de adaptar a los diversos contextos de aplicación (Chile, Universidad de Concepción), con un total de 71 ítems. La muestra piloto quedó integrada por 47 docentes de las diversas facultades de ciencias y humanidades de la Universidad de Concepción.

Asimismo, Echeverry (2013) ha publicado un modelo teórico sobre EdE de profesores de educación superior tomando en consideración una visión integral sustentada en tres saberes fundamentales para el desarrollo de la práctica docente, por un lado la dimensión del ser, por otra la del saber-saber y finalmente la del saber-hacer, estableciendo varios perfiles de EdE que son los siguientes:

- Constructivo-Humanista Emotivo: caracterizado por el desarrollo de habilidades para la vida, permitiendo a los alumnos ser mejores seres humanos. Son docentes que parecen tener una amplia experiencia docente y demuestran pasión por su profesión y por la disciplina que enseñan. Tienen pasión por construir mensajes y transmitir información hace que las emociones, afectos y sentimientos influyan en las formas de enseñar y de aprender determinados contenidos por parte de su alumnado, determinados contenidos (Zabalza, 2006; Day, 2006).

- Constructivo-Profesional Entusiasta: se centra en el desarrollo de las competencias profesionales que permite a los estudiantes elaborar su propio conocimiento adaptado a la realidad profesional. Son docentes joviales y entusiastas que transmiten tranquilidad y cercanía a su alumnado. Suelen estar vinculados al ámbito científico y sus discursos son claros y específicos promoviendo la construcción y comprensión de los contenidos disciplinares.

- Disciplinar-Experto Sereno: son docentes con poca trayectoria profesional que profundizan en un campo teórico determinado. Suelen estar vinculados al ámbito humanístico y tener poca expresividad gestual y corporal. No favorecen la participación del alumnado, que es pasivo, y metodológicamente son sistemáticos.

- Constructivo-Social Laissez-Faire: se promueve la FP y la autonomía social de los estudiantes. Los docentes buscan fomentar la reflexión y la construcción de la autoestima entre el alumnado para mejorar la convivencia. Suelen estar ligados al ámbito científico y ofrecen estrategias al alumnado para favorecer su aprendizaje.

- Ecléctico-Estructurado: el profesorado con este estilo favorece en el alumnado las relaciones sociales para la adquisición de contenidos disciplinares integrados en el esquema mental de cada estudiante.

En estas últimas investigaciones sobre los $\mathrm{EdE}$, se hace evidente, uno de los problemas más acusados en el campo de la investigación educativa y más concretamente, en algunos estudios de los EdA y los EdE: el tamaño de la muestra. En este caso, una muestra reducida para un instrumento dicotómico impide un análisis estadístico exhaustivo, que permita darle un constructo y una validez sólida al instrumento. Muestras tan pequeñas han derivado que el índice de fiabilidad del cuestionario de EdE, como el de Chiang et al. (2013), no aparezca referenciado, y que el instrumento se transforme en una escala tipo Likert (Martínez-Geijo, 2002) o bien que la investigación se reconvierta en un estudio de casos impiden la aplicación y generalización del instrumento en otras poblaciones de estudio. Este problema, ya enunciado por Bennett, es al que se intenta dar solución en este planteamiento, con la finalidad de lograr 
construir un marco válido y fiable que permita su difusión y aplicación en otros ámbitos científicos y profesionales tal y como se ha reflejado en la publicación de Renés-Arellano y Martínez-Geijo (2015).

\subsection{Modelo y cuestionario sobre EdE desde un enfoque cognitivo- constructivista}

En este trabajo se han descrito diversos instrumentos de evaluación y diagnóstico de los EdA y los EdE del alumnado y profesorado en diversos ámbitos educativos. En este sentido, tal y como se ha mencionado anteriormente, se ha querido dar respuesta a la necesidad actual de responder pedagógicamente a las necesidades del aula. Sin embargo, esto no puede realizarse sin antes analizar el contexto en el que se circunscribe la actividad educativa. Conocer las maneras de aprender y de enseñar del alumnado y profesorado, permite responder a esta necesidad. Por dicho motivo, en este estudio se ha intentado romper con los estereotipos señalados por Bennett en cuanto al problema del tamaño de la muestra y la validez de un instrumento que identifique los EdE, se ha creado el CEdE (Cuestionario de Estilos de Enseñanza, Renés-Arellano, 2014) tomando como referencia el publicado por Martínez-Geijo (2002) y el CHAEA.

Los EdE identificados en el cuestionario son los compartidos con Martínez-Geijo (2007) y Renés et al. (2013): Abierto, Formal, Estructurado y Funcional, correspondientes a los EdA de Alonso, Gallego y Honey (2012): Activo, Reflexivo, Teórico y Pragmático, respectivamente (véase figura 2).

\begin{tabular}{cc}
\hline EdA según el CHAEA & EdE según el CEE \\
\hline Activo & Activo \\
\hline Reflexivo & Formal \\
\hline Teórico & Estructurado \\
\hline Pragmático & Funcional \\
\hline
\end{tabular}

Figura 2. Relación de EdE según el CHAEA y EdA según el CEdE

Destacar que el CEdE (Cuestionario de EdE) ha sido diseñado con el mismo número de ítems que el CHAEA, un total de 80 , distribuidos aleatoriamente según cada uno de los EdE y con una respuesta dicotómica, aspectos empíricos que se desarrollarán con posterioridad. Asimismo, las características propias de cada EdE y su relación con el EdA son las siguientes se comparten con las de Martínez-Geijo (2007):

\section{- EdE Abierto}

En el EdE Abierto se encuentran aquellos profesores que con sus comportamientos docentes favorecen al alumnado del EdE con preferencia ALTA o MUY ALTA, a los estudiantes con EdE ACTIVO. 
El profesorado de este EdE se plantea con frecuencia nuevos contenidos, aunque no estén incluidos en el programa, lo que significa que no se ajustan de manera estricta a la planificación. Motivan a los estudiantes con actividades novedosas, con problemas reales del entorno, y los animan a ser originales en la búsqueda de soluciones a las tareas planteadas.

Promueven el trabajo en equipo y la generación de ideas por parte del estudiante sin ninguna limitación formal. Permiten que se debatan las cuestiones y argumentos que se plantean en el aula dejando que los alumnos actúen de forma espontánea. Suelen cambiar con frecuencia de metodología. Utilizan las simulaciones, dramatizaciones y otras estrategias didácticas de carácter abierto, de manera que en la clase se asuman roles y se realicen presentaciones, intervenciones no preparadas, debates y otras actividades que hagan del aula un espacio dinámico. Procuran que los estudiantes no trabajen mucho tiempo sobre la misma actividad, para lo que plantean varias tareas a la vez y permiten flexibilidad en la temporalización y el orden de realización.

Anuncian las evaluaciones con poca anticipación, las cuales, por lo general, constan de pocas preguntas y respuestas abiertas, no concediendo demasiada importancia a la presentación, los detalles y el orden. Se inclinan por los estudiantes y colegas/compañeros con ideas originales, espontáneos, participativos e inquietos. Son partidarios de romper las rutinas, de transmitir su estado de ánimo y trabajar en equipo con otros docentes. Suelen estar bien informados de la actualidad. Son activos, creativos, improvisadores, innovadores, flexibles y espontáneos.

\section{- EdE Formal}

En el EdE Formal se encuentran aquellos profesores que con sus comportamientos docentes favorecen al alumnado del EdE con preferencia ALTA o MUY ALTA, a los estudiantes con EdE REFLEXIVO.

Los docentes de este EdE son partidarios de la planificación detallada de su enseñanza y lo comunican a sus alumnos. Se rigen estrictamente por lo planificado. No admiten la improvisación y no suelen impartir contenidos que no estén incluidos en el programa. Tienden a abordar la enseñanza con explicaciones y actividades diseñadas con detalle, profundidad, analizando el contenido desde diferentes perspectivas, sin importarles el tiempo, aunque teniendo como referente su programación.

Fomentan y valoran en los estudiantes la reflexión y el análisis desde la racionalidad, dejando tiempo para las revisiones y repasos. Promueven el trabajo individual sobre el grupal con estrategias metodológicas donde las funciones y los roles vienen delimitados para que los estudiantes no actúen de forma improvisada y conozcan en cada momento su quehacer. Anuncian las fechas de los exámenes o controles con suficiente anticipación. Se inclinan por los estudiantes tranquilos, reflexivos, ordenados y metódicos. No son partidarios del trabajo en equipo con otros docentes y si lo hacen prefieren que se les asigne la parte de la tarea a desarrollar. Les afecta las opiniones que se tiene de ellos y el temor a quedar en inferioridad en cuanto a las expectativas que despiertan. Son responsables, reflexivos, cuidadosos, tranquilos y muy pacientes.

\section{- EdE Estructurado}


En el EdE Estructurado se encuentran aquellos profesores que con sus comportamientos docentes favorecen al alumnado del EdE con preferencia ALTA o MUY ALTA, a los estudiantes con EdE TEÓRICO.

Los docentes con este EdE otorgan bastante importancia a la planificación y ponen énfasis en que sea coherente, estructurada y bien presentada. Tienden a impartir los contenidos integrados siempre en un marco teórico amplio, articulado y sistemático. La dinámica de la clase suele desarrollarse bajo una cierta presión, evitando cambiar por frecuencia de metodología. Las actividades a trabajar son preferentemente complejas.

Aunque no son partidarios del trabajo en equipo entre los estudiantes, cuando lo hacen favorecen que los agrupamientos sean homogéneos intelectualmente 0 por calificaciones. Inciden en mantener un clima de aula ordenado y tranquilo. No dan opción a la espontaneidad, la ambigüedad ni a respuestas no razonadas. En las evaluaciones solicitan a los alumnos que los ejercicios/preguntas los resuelvan/contesten especificando y explicando cada paso. En la relación y trabajo con otros docentes, casi siempre cuestionan las temáticas que se tratan, procurando ser los últimos en dar sus opiniones. Dentro de este estilo se encuentran los docentes que se caracterizan por ser objetivos, lógicos, perfeccionistas y sistemáticos.

\section{- EdE Funcional}

En el EdE Abierto se encuentran aquellos profesores que con sus comportamientos docentes favorecen al alumnado del EdE con preferencia ALTA o MUY ALTA, a los estudiantes con EdE PRAGMÁTICO.

Los docentes con este EdE siendo partidarios de la planificación, ponen el énfasis en su viabilidad, funcionalidad y concreción. Su preocupación es cómo llevarla a la práctica. Otorgan mayor valor a los contenidos procedimentales y prácticos que a los teóricos. En las explicaciones sobre contenidos teóricos, siempre incluyen ejemplos prácticos y frecuentemente tomados de la vida cotidiana.

En la dinámica de la clase no emplean mucho tiempo en las exposiciones teóricas o magistrales, sustituyéndolas por experiencias y trabajos prácticos. Pueden acudir al aula con personas expertas en determinada materia para explicar ante la clase cómo se realizan algunas actividades. Con el alumnado son partidarios del trabajo en equipo, dándoles las instrucciones lo más claras y precisas posibles para el desarrollo de la tarea. Se inclinan por los estudiantes prácticos, realistas, curiosos, emprendedores y siempre amantes de las experiencias prácticas que tengan utilidad. En la relación y trabajo con otros docentes se implican en todo aquello que les sea útil tanto en lo personal como en lo profesional. En las reuniones de trabajo suelen insistir una y otra vez en que no se divague y se concrete.

Una vez descritas las características propias de cada EdE, se procedió a establecer categorías o dimensiones que sirviesen para construir el instrumento posterior de diagnóstico, identificándose un total de nueve, a las que se asignó cada uno de los ítems. A continuación se muestran las dimensiones del instrumento definitivo. 
Planificación: está relacionada con la organización temporal, espacial, conceptual y social que realiza el docente en el aula. Cuál es la estructura de la asignatura, selección y distribución de las actividades, programación de aula...

Intervención de los alumnos en clase: vinculada a la metodología que se aplica en el aula, al tipo de enseñanza en cuanto a participación del alumnado, tipo de explicaciones, relevancia de contenidos, valoración del trabajo de los estudiantes...

Dinámica de clase: está relaciona con la organización grupal del alumnado en clase, trabajo en equipo o individual, tipos de agrupamiento (pequeño, mediano, grande, por niveles académicos...), dinamismo metodológico, combinación teoría y práctica...

Pruebas de evaluación: en esta dimensión se valora el tipo de preguntas que se realiza al alumnado (abiertas, cerradas o mixtas), la valoración de la presentación de trabajos, orden, limpieza, coherencia y cohesión en las respuestas y las cuestiones prácticas.

Contenidos: se tiene presente la contextualización, las preguntas, la profundidad o superficialidad y la practicidad de los contenidos a impartir en el aula.

Actividades: la reiteración, creatividad, variedad temática, la búsqueda de información, la estructura o la relación de ideas, son algunas de las características que se abordan en esta dimensión.

Relaciones con otros compañeros: la capacidad de escucha, de animar en el trabajo, el razonamiento, las relaciones profesionales, la objetividad y la claridad son considerados elementos de esta categoría.

Alumnos: la tipología del alumnado en cuanto a sus características espontáneas, inquietos, reflexivos, tranquilos, prácticos, coherentes, realistas hacen que el docente prefiera un colectivo u otro.

Personales: la manera de expresar cómo se siente el docente, si es capaz de reflejar su estado de ánimo, si se agobia ante diversas situaciones, si es metódico, analítico, individualista, práctico, emocional o concreto, determina el tipo de EdE del profesorado.

En la siguiente tabla 3 se exponen el total de ítems del cuestionario estructurados según las dimensiones de análisis con palabras clave de la redacción de cada una de las preguntas (Renés-Arellano y Martínez-Geijo, 2015):

EdE en función de los EdA en relación con las dimensiones del proceso de enseñanza EdE que favorecerían el EdA

$\begin{array}{llcl}\text { Activo-Abierto } & \text { Reflexivo-Formal } & \begin{array}{c}\text { Teórico- } \\ \text { Estructurado }\end{array} & \begin{array}{c}\text { Pragmático- } \\ \text { Funcional }\end{array} \\ \end{array}$




\begin{tabular}{|c|c|c|c|c|c|c|c|c|}
\hline 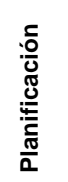 & $\begin{array}{r}1 . \\
47 .\end{array}$ & $\begin{array}{l}\text { Me limita } \\
\text { No comunicar a los } \\
\text { alumnos }\end{array}$ & 36. & $\begin{array}{l}\text { Es previa al inicio } \\
\text { del curso } \\
\text { Es presentada al } \\
\text { alumnado }\end{array}$ & 69. & Coherente y lógica & $\begin{array}{l}80 . \\
61 .\end{array}$ & $\begin{array}{l}\text { Viable } \\
\text { Práctica }\end{array}$ \\
\hline 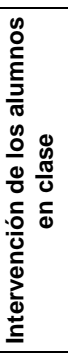 & $\begin{array}{l}48 . \\
78 .\end{array}$ & $\begin{array}{l}\text { Pedir voluntarios } \\
\text { Explicaciones } \\
\text { breves }\end{array}$ & $\begin{array}{l}24 . \\
12 .\end{array}$ & $\begin{array}{l}\text { Pregunto si he } \\
\text { avisado para que } \\
\text { lo preparen } \\
\text { Solo pregunto si } \\
\text { he avisado } \\
\text { Animo a pensar } \\
\text { antes de hablar }\end{array}$ & 44. & $\begin{array}{l}\text { Aula ordenada, sin } \\
\text { espontaneidad } \\
\text { Intervención lógica y } \\
\text { coherente }\end{array}$ & 9. & $\begin{array}{l}\text { Reconozco su } \\
\text { mérito }\end{array}$ \\
\hline 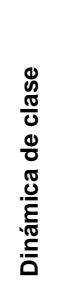 & $\begin{array}{l}75 . \\
65 .\end{array}$ & $\begin{array}{l}\text { Trabajo en equipo } \\
\text { Pregunto sin avisar } \\
\text { Metodología } \\
\text { variada }\end{array}$ & 2. & $\begin{array}{l}\text { No trabajo en } \\
\text { equipo } \\
\text { Pocos temas pero } \\
\text { en profundidad } \\
\text { Explicación en } \\
\text { profundidad }\end{array}$ & $\begin{array}{l}10 . \\
27 . \\
21 . \\
15 .\end{array}$ & $\begin{array}{l}\text { Sobre preguntas y } \\
\text { debates } \\
\text { Agrupo por nivel } \\
\text { Rutinaria } \\
\text { Trabajo con presión }\end{array}$ & $\begin{array}{l}34 . \\
13 .\end{array}$ & $\begin{array}{l}\text { Poca teoría } \\
\text { Recurro a } \\
\text { expertos } \\
\text { Explicaciones con } \\
\text { ejemplos }\end{array}$ \\
\hline 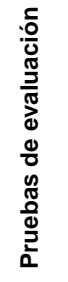 & $\begin{array}{l}68 . \\
79 .\end{array}$ & $\begin{array}{l}\text { Preguntas abiertas } \\
\text { No valora } \\
\text { presentación y } \\
\text { orden }\end{array}$ & 28. & $\begin{array}{l}\text { Valoro orden y } \\
\text { presentación } \\
\text { Anunciadas con } \\
\text { antelación }\end{array}$ & 40. & $\begin{array}{l}\text { Respuestas lógicas y } \\
\text { coherentes } \\
\text { Valoro el proceso de } \\
\text { respuesta }\end{array}$ & 57. & $\begin{array}{l}\text { Respuestas } \\
\text { concretas } \\
\text { Cuestiones } \\
\text { prácticas }\end{array}$ \\
\hline 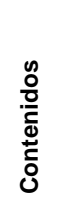 & $\begin{array}{r}7 . \\
11 .\end{array}$ & $\begin{array}{l}\text { Priorizo las } \\
\text { preguntas } \\
\text { espontáneas } \\
\text { Sin profundizar }\end{array}$ & 16. & $\begin{array}{l}\text { Priorizo lo } \\
\text { planificado } \\
\text { Explicaciones sin } \\
\text { importar el tiempo }\end{array}$ & $\begin{array}{l}58 . \\
35 .\end{array}$ & $\begin{array}{l}\text { Contextualizados } \\
\text { Priorizo lo teórico }\end{array}$ & 66. & Prácticos \\
\hline$\frac{d}{\frac{0}{0}}$ & $\begin{array}{l}26 . \\
31 . \\
60 .\end{array}$ & $\begin{array}{l}\text { Son no repetitivas } \\
\text { Inventar temas } \\
\text { Favorezco la } \\
\text { creatividad }\end{array}$ & $\begin{array}{l}3 . \\
33 . \\
45 .\end{array}$ & $\begin{array}{l}\text { Dejo tiempo } \\
\text { necesario } \\
\text { Sin creatividad } \\
\text { De búsqueda de } \\
\text { información }\end{array}$ & $\begin{array}{r}6 . \\
14 .\end{array}$ & $\begin{array}{l}\text { Bien estructuradas } \\
\text { De relacionar, asociar o } \\
\text { categorizar } \\
\text { Complejas con } \\
\text { pasos a seguir }\end{array}$ & $\begin{array}{l}37 . \\
29 . \\
42 .\end{array}$ & $\begin{array}{l}\text { Orientadas } \\
\text { Realistas } \\
\text { Sustentadas en lo } \\
\text { práctico } \\
\text { Prácticas } \\
\text { Valoro la solución } \\
\text { sobre el proceso }\end{array}$ \\
\hline 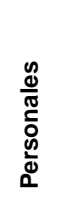 & $\begin{array}{l}43 . \\
56 .\end{array}$ & $\begin{array}{l}\text { Reflejo estado de } \\
\text { ánimo } \\
\text { No me agobio y } \\
\text { replanteo } \\
\text { Evito ser metódico }\end{array}$ & 55. & $\begin{array}{l}\text { Análisis en exceso } \\
\text { Trabajo individual }\end{array}$ & $\begin{array}{l}53 . \\
32 .\end{array}$ & $\begin{array}{l}\text { Cuestiono casi todo } \\
\text { Me disgusta que noten } \\
\text { falta de conocimiento }\end{array}$ & 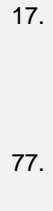 & $\begin{array}{l}\text { Práctico y útil } \\
\text { sobre lo } \\
\text { emocional } \\
\text { Me gusta lo } \\
\text { concreto }\end{array}$ \\
\hline 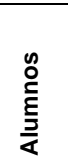 & 18. & $\begin{array}{l}\text { Les prefiero } \\
\text { espontáneos e } \\
\text { inquietos }\end{array}$ & 41. & $\begin{array}{l}\text { Les prefiero } \\
\text { reflexivos, } \\
\text { tranquilos y } \\
\text { metódicos }\end{array}$ & 74. & $\begin{array}{l}\text { Preferencia por actitud } \\
\text { coherente }\end{array}$ & 50. & $\begin{array}{l}\text { Les prefiero } \\
\text { prácticos y } \\
\text { realistas }\end{array}$ \\
\hline
\end{tabular}




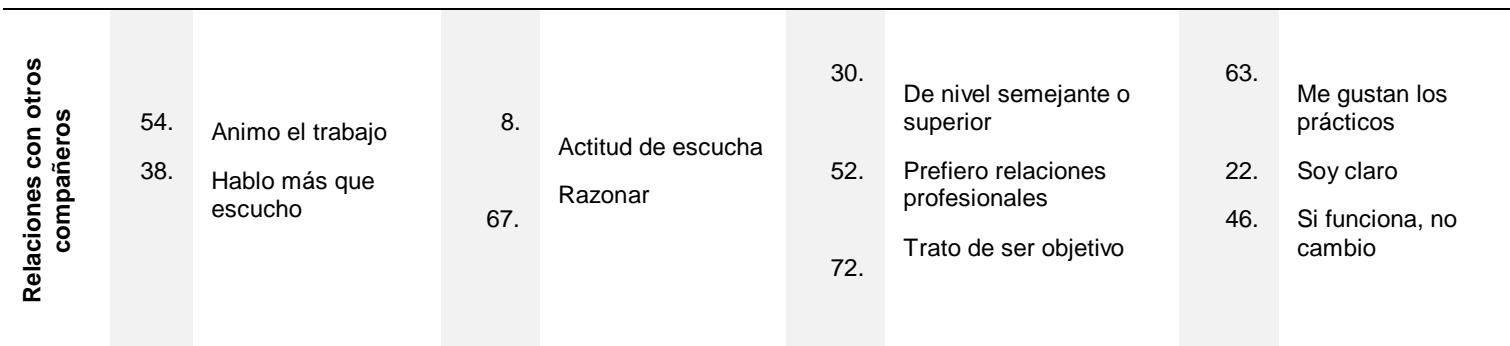

Tabla 3. Estructura de los ítems del CEdE según las dimensiones del cuestionario y los EdA

\section{CONCLUSIÓN}

Los procesos de enseñanza y aprendizaje han guiado este trabajo hacia la búsqueda de información de los orígenes del elemento pedagógico y vertebrador del sistema educativo, social y cultural: enseñar y aprender. Se detectó la necesidad de avanzar en el estudio de las maneras de aprender del alumnado y de aprender y enseñar del profesorado, un campo aún por descubrir y que ayudará en la mejora de los procesos educativos y en la búsqueda de un mejor rendimiento académico del alumnado. Por dicho motivo, se seleccionó un modelo de análisis sustentando en las maneras de aprender, el instrumento de medida CHAEA. Es un cuestionario que permite identificar los EdA de las personas (Activo, Reflexivo, Teórico y Pragmático) y que por su rigurosidad, validez, divulgación y prestigio en el panorama científico, se ha arraigado como instrumento útil, fácil y eficaz entre el colectivo docente para su utilización con el alumnado.

Ante esta situación y la variabilidad de EdA del alumnado, diversos autores han avanzado en la búsqueda pedagógica por entender no solamente las maneras de aprender sino también, cómo enseña el profesorado, que es el foco de interés de este capítulo. De la mano de Bennett, cuyos estudios han generado el surgimiento de este trabajo, se identificaron dos EdE: tradicional y progresista. Posteriormente, otras investigaciones han ido tomando relevancia en el estudio de las formas de enseñar, destacando la conceptualización del modelo de Martínez-Geijo (2002, 2011) quien planteó una investigación sobre EdE sustentada en el enfoque cognitivo-constructivista para categorizar los comportamientos de enseñanza, siguiendo el patrón de Bennett, y el enfoque cuaternario que presentó Alonso, Gallego y Honey (2012) sobre los EdA, en la misma línea que Honey y Mumford (1992), Kolb (1984) y Gregorc (1984). Realizada una aproximación teórica de la enseñanza, su etimología, enfoques, modelos e instrumentos relacionados con los $\mathrm{EdE}$, se ha diseñado un cuestionario respondiendo a la estructura cuaternaria y tipo de respuesta dicotómica del CHAEA, e identificando cada EdA con un EdE. De tal modo que se obtiene una relación entre la manera de aprender del alumnado y la forma de enseñar del profesorado, para posteriormente, ofrecer alternativas de trabajo metodológico.

Los EdE que se extraen de este trabajo son el Abierto que se corresponde con el EdA Activo, el Formal con el EdA Reflexivo, el Estructurado con el EdA Teórico y el Funcional con el EdA Pragmático. Identificados cada uno de ellos y descritos con sus correspondientes características se procedió a realizar las aportaciones vinculadas a las implicaciones pedagógicas de cada 
EdE en función del EdA, con la finalidad de encontrar actividades y metodologías que se adecúen mejor a las formas de aprender del alumnado y con ello, se fomente el rendimiento académico. $Y$ es que, si el docente fuese capaz de adecuar sus EdE a los EdA de su alumnado, planteando actividades diversas adaptadas a dichos estilos, el rendimiento académico, tal y como se recoge anteriormente, podría mejorar, y por ende, la calidad docente.

La elaboración de un cuestionario para diagnosticar los EdE, no tiene una finalidad etiquetadora ni determinante en su aplicación educativa sino que subyace de éste una serie de estilos que permitirán al docente conocerse a sí mismo y buscar aquellas estrategias metodológicas que mejor se adecúen a la realidad profesional en la que trabaja. Además, en este caso, el empleo del CEdE junto con el CHAEA para profesores para ambos colectivos, docentes y discentes, permite identificar cómo aprende el alumnado y cómo aprende y enseña el profesorado. Ayuda al docente a conocer cómo aprende y a descubrir si su manera de enseñar es equitativa a su manera de aprender, o por el contrario es capaz, independientemente de su manera de aprender, de adaptarse a través de EdE variados, a la diversidad de EdA de su alumnado, y por consiguiente favorecer la mejora del rendimiento académico. Finalmente, se considera que la incorporación de los EdA y EdE requiere de reflexiones en torno a los siguientes parámetros:

1) La realidad sociocultural de los agentes educativos de la institución en la que trabaje, considerando el contexto en el que se enmarca el centro, su profesorado, alumnado y familias.

2) En la praxis educativa diaria es imprescindible tener en cuenta las características del alumnado de su aula-grupo, especialmente sus EdA, con la finalidad de responder pedagógicamente a las necesidades reales.

3) Elección de metodologías adecuadas a cada grupo-clase, seleccionando los objetivos, contenidos, actividades, recursos y evaluación que responda a la programación didáctica en función de la realidad de su alumnado. Incorporando aquellas estrategias, instrumentos de desarrollo, diagnóstico y/o evaluación que favorezcan una adecuada respuesta pedagógica en su práctica docente, en la que el CHAEA y el CEdE, entre otros, pueden servir de referentes para organizar, seleccionar y definir su labor profesional ayudándole a conocer mejor a su alumnado (la manera de aprender), y a sí mismo (su manera de aprender y de enseñar).

Por todo ello, se considera fundamental tener presente el enfoque de enseñanza que se adopte en el aula, que el profesorado sea capaz de racionalizar la heterogeneidad de su alumnado y adopte comportamientos de enseñanza, adecuados a los EdA de su alumnado. Porque "formar a un profesor es, más bien establecer las premisas sobre las cuales éste debe basar el razonamiento práctico acerca de la enseñanza en situaciones específicas" (Shulman, 1989:46). Para ello resulta sustancial, previamente, conocer los propios EdA y EdE, independientemente del nivel educativo en el que se desarrolle la labor docente. Porque "el conocimiento y la atención a los EdA en 
la práctica de aula pueden contribuir a un mejor ajuste entre cómo se aprende y cómo se enseña" (Martínez-Geijo, 2008:92).

\section{REFERENCIAS}

Alonso, C. M., Gallego, D. J. y Honey, P. (2012). Los estilos de aprendizaje. Procedimientos de Diagnóstico y Mejora. Ediciones Mensajero: Bilbao.

Arends, R. (2007). Aprender a enseñar. México: McGraw-Hill.

Bennett, N. (1979). Estilos de enseñanza y progreso de los alumnos. Madrid: Morata.

Biggs, J., Kermber, D. y Leurng, D. (2001). The revised two-factor study process questionnaire: R-SPQ-2F. British journal of educational psychology, 71, 133-149. https://doi.org/10.1348/000709901158433

Bransford, J. et al. (2000). How People Learn. Washington: National Academies Press.

Chiang, M. T., Díaz-Larenas, C., Rivas-Aguilera, A. y Martínez-Geijo, P. (2013). Validación del cuestionario Estilos de Enseñanza (CEE). Un instrumento para el docente de educación superior. Revista Estilos de Aprendizaje, $12(11), 1-16$.

Colén, M. T., Giné, N. e Imbernon, F. (2006). La carpeta de aprendizaje del alumnado universitario. La autonomía de estudiante en el proceso de aprendizaje. Barcelona: Octaedro.

Contreras, O. (1998). Didáctica de la Educación Física. Madrid: McGraw-Hill.

Day, C. (2006). Pasión por Enseñar. La identidad profesional y personal del docente y sus valores. Madrid: Narcea.

Díaz-Barriga, F. y Hernández-Rojas, G. (2002). Estrategias docentes para un aprendizaje significativo. Una interpretación constructivista. Madrid: Mc GrawHill.

Echeverry, L. M. (2013). Los estilos de enseñanza de los profesores de Educación Superior: Una mirada desde la consideración de un modelo integral. (Tesis de maestría) Universidad de Antioquia, Medellín.

Freire, P. (1979). Educación y acción cultural. Bilbao: Zero.

Fuentes, C. y Espinoza, P. (2014). Influencia de los estilos de enseñanza y los estilos de aprendizaje en el rendimiento académico en una muestra de los estudiantes de la carrera de pedagogía, ciencias naturales y química. Una mirada desde la transposición didáctica. (Tesis de maestría). Universidad de Concepción, Chile.

Gargallo, B. et al. (2010, Noviembre). Modelos de enseñanza y aprendizaje en la universidad. Ponencia presentada al XXIX Seminario Interuniversitario de Teoría de la Educación: Formación y participación de los estudiantes en la universidad. Madrid, España. 
González-Pérez, T. (1993). La figura del maestro en la historia del pensamiento pedagógico. Revista interuniversitaria de formación del profesorado, 16, 135-144.

Gimeno, J. y Pérez-Gómez, A. (1998). Comprender y transformar la enseñanza. Madrid: Morata.

González-Peiteado, M., López-Castedo, A. y Pino-Juste, M. (2013). Análisis psicométrico de una escala sobre estilos de enseñanza (ESEE). Enseñanza \& Teaching, 31 (1), 181-198.

Gregorc, A. (1984). Gregorc Style Delineator. Columbia, CT: Gregorc Associates, Inc.

Gros, B. (1997). Diseños y programas educativos. Pautas pedagógicas para la elaboración de software. Barcelona: Ariel.

Haidt, R.C. (2000). Curso de didática geral. São Paulo: Ática.

Hervás, M. A. (2003). Estilos de enseñanza y aprendizaje en escenarios educativos. Granada: Grupo editorial universitario.

Honey, P. y Mumford, A. (1992). Questions and answers on Learning Styles Questionnaire. Industrial and Commercial Training, 24 (7), 10-13.

Isaza, L. y Henao, G. C. (2012). Actitudes-Estilos de enseñanza: su relación con el rendimiento académico. International Journal of Psychological Research, 5 (1), 133-141.

Kolb, D. (1984). Experiential learning. Experience as the source of learning and development. New Jersey: Prentice-Hall.

Manterola, C. (2002). Enseñar a Enseñar. Escuela de educación. Venezuela: Universidad Central de Venezuela.

Martínez-Geijo, P. (2002). Categorización de comportamientos de enseñanza desde un enfoque centrado en los estilos de aprendizaje. (Tesis de maestría). UNED, España.

Martínez-Geijo, P. (2008). Estilos de aprendizaje: pautas metodológicas para trabajar en el aula. Revista Complutense de Educación, 19 (1), 77-94.

Martínez-Geijo, P. (2007). Aprender y enseñar. Los estilos de aprendizaje y de enseñanza desde la práctica de aula. Bilbao: Mensajero.

Martínez-Geijo, P. (2011). De los estilos de aprendizaje a los estilos de enseñanza _ un paso hacia adelante en el ajuste entre aprender y enseñar. En D. Gallego y C. Alonso, Innovación y gestión del talento. Desarrollo de conocimiento y aprendizaje desde la perspectiva educativa (pp. 403-416). Cáceres: EBS.

Nortes-Martínez, R. y Nortes-Checa, A. (2012). Enseñanza, aprendizaje y evaluación en el Grado de Maestro de Primaria. Educatio siglo XXI, 30 (2), 289-312.

Renés, P., Echeverry, L. M., Chiang, M. T., Rangel, L. y Martínez-Geijo, P. (2013). Estilos de enseñanza: un paso adelante en su conceptualización y diagnóstico. Revista Estilos de Aprendizaje, 11 (1), 4-8. 
Renés-Arellano, P. y Martínez-Geijo, P. (2015). Estilos de enseñanza y aprendizaje. Conceptualizaciones, investigaciones y orientaciones para la práctica. Bilbao: Mensajero.

Renés-Arellano, P. y Martínez-Geijo, P. (2016). Una mirada a los estilos de enseñanza en función de los estilos de aprendizaje. Journal of Learning Styles, 9 (18), 224-243.

Salinas, J. y Urbina, R. (2006). Bases para el diseño, la producción y la evaluación de procesos de Enseñanza-Aprendizaje mediante nuevas tecnologías. En J. Cabero (coord.). Nuevas Tecnologías aplicadas a la Educación (pp. 41-75). Madrid: La Crujía.

Salvador, L., Argos, E., Ezquerra, Osoro, J. y Castro, A. (2011). Perfiles de estilos de aprendizaje de los estudiantes universitarios y metodologías docentes. Revista Bordón, 63 (2), 41-52.

Sánchez-Ortega, J. (2011). Diagnóstico y aplicación de los estilos de aprendizaje en los estudiantes del bachillerato internacional: una propuesta pedagógica para la enseñanza eficaz de la robótica educativa. (Tesis de maestría). UNED, España.

Shulman, L. S. (1989). Paradigmas y programas de investigación en el estudio de la enseñanza: una perspectiva contemporánea. En M. C. Wittrock (coord.) La investigación de la enseñanza, I. Enfoques, teorías y métodos (9-91). Barcelona: Paidós.

Zabalza, M. (2006). Competencias docentes del profesorado universitario. Calidad y desarrollo profesional. Madrid: Narcea. 
